\title{
Fatigue of Sandwich Composites in Air and Seawater
}

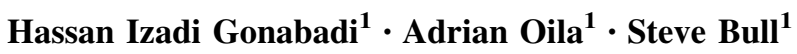

Received: 23 December 2015/Revised: 16 March 2016/Accepted: 18 March 2016/Published online: 5 April 2016

(C) The Author(s) 2016. This article is published with open access at Springerlink.com

\begin{abstract}
In this study, the fatigue behaviour of sandwich composites was investigated in both, air and seawater. $S-$ $N$ curves were obtained for different stress levels. 3-point bending (3PB) tests were also performed to investigate strength degradation during the fatigue test. Finite element analyses were carried out to determine the stress distribution in the test coupons used in bending tests. These were used to predict the failure of the composite and to interpret the experimental results. There is good agreement between finite element analysis and experimental results at lower loads. Small deviations were observed at higher loads due to the influence of defects. The typical failure modes observed under quasi-static 3PBT were debonding, fibre pull-out and matrix cracking.
\end{abstract}

Keywords Sandwich composites $\cdot$ Fatigue $\cdot$ Seawater

\section{Introduction}

Due to their light weight and their relatively, high specific modulus and strength composite materials are ideal candidates in various applications such as in marine energy conversion systems. The tidal turbine technology is not very well matured and experimental data are needed to aid in material selection [1-4]. In order to use composite materials in such applications, knowledge of their mechanical behaviour is required and also a better

Adrian Oila

adrian.oila@newcastle.ac.uk

1 School of Chemical Engineering and Advanced Materials, Newcastle University, Newcastle upon Tyne NE1 7RU, UK understanding of the different failure modes under various loading conditions is essential.

Sandwich composites are multi-layered materials used as a single structure which is divided in three main constituents: two external thin and stiff face sheets and a central soft and thick core mat. The face sheets are bonded to the core mat to allow the transfer of loads between constituents [2]. In sandwich composites, by placing the stiff material between the faces far from the neutral axes, a high bending stiffness is obtainable. The faces endure most of the normal and bending stresses, while the core carries the shear stresses $[3,5]$.

Global factors were considered for the evaluation of mechanical behaviour of sandwich composites [1]; however, the results are not very precise since there is no comparison made between experimental and analytical methods. Davalos and Qiao [6] performed finite element analysis on honeycomb composites and reached the conclusion that there is a good agreement between experimental values and analytical prediction in the four point bending test. Mechanical behaviour of a glass-polyester sandwich structures for high performance equipment in marine applications was investigated by Di Bella [3]. Behaviour of the sandwich composites was studied under different quasi-static conditions like three point bending, flatwise, edgewise compression and torsion tests. It was found that a new laminate sequence can improve the properties of sandwich structures. It was shown that from the aforementioned static mechanical tests and failure analysis the use of a binder at the interface sheet/core mat can improve the mechanical properties and reduce crack initiation.

In a general review [7] of sandwich composite materials assessed by flexural tests, the effect of material variables such as span to thickness ratio and specimen flaws such as 
voids and trapped air is discussed and it was found that in sandwich composites these variables may also affect failure modes. In three point bending test due to the applied force, the test specimen is divided in two sections, the upper section which experiences compressive stresses and the lower section where tensile stresses occur. Consequently in the concave and convex side of test specimens corresponding to compressive and tensile stressed regions, different potential failure modes during a flexural test may occur. Theoretically, the most widely used failure criterion for composite materials is the maximum stress failure criterion which predicts that the material will fail once the magnitude of the stress in any direction exceeds its permissible level in that direction. The advantage of using this failure criterion is that it discerns the particular mechanism of failure within a ply. In three point bending, six principal stresses are developed and when each of these stresses exceeds its relevant tensile, compressive and shear strength, the failure modes are tension, compression and inplane shear, respectively [8]. The experimental requirements for three point bending test (3PBT) on composites are simpler than those for a tensile or compressive tests, as the influence of defects and geometrical stress concentration sites on the composite specimens is less severe. Moreover, a parallel sided specimen can be used which is simple to prepare.

The main objective of our work was to compare the fatigue life of sandwich composites in air and in seawater and to estimate the magnitude of the difference.

\section{Experimental}

\subsection{Materials}

In this study, three different configurations of face sheet and core mat thickness were used (see Fig. 1). PVC foam was used as a core material and chopped strand mat (CSM) glass in the matrix of polyester resin was used in the face sheet material. The glass fibres are randomly distributed in the matrix. The test specimens for 3PBT are flat rectangular beams with a constant cross-sectional area. During the 3PBT, the specimen is supported on two rollers and the load is applied at the centre of the specimen on the opposite
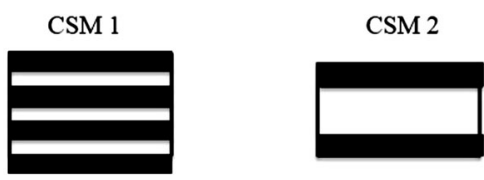

$\mathrm{CSM} 3$

Fig. 1 The structural sections of the test beams: dark and white area represent face sheet (CSM material) and PVC foam (core material), respectively side. The dimensions of the test specimens are specified in the standard ES BN ISO 14125 [9]. Three different configurations of skin and core mat thickness were used as listed in Table 1.

\subsection{Mechanical Testing}

In order to determine the required parameters for numerical simulation, composite specimens were subjected to 3PBT. The 3PBT was performed using a universal mechanical testing machine at a speed of $2 \mathrm{~mm} / \mathrm{min}$, according to the ASTM C393-00 for sandwich composite structures. The specimens were mounted in the three point bending grips with span to thickness ratio of 20. All rolls have the same size ( $2 \mathrm{~cm}$ in diameter) and the span between two supporting rolls was $100 \mathrm{~mm}$. For sandwich composite structures the face sheet bending stress $(\delta)$, the core shear stress

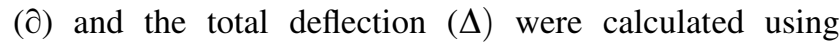
Eqs. (1)-(4).

$\delta=\frac{P L}{2 t(h+c) b}$

$\partial=\frac{P}{(h+c) b}$

$\Delta=\frac{P L^{3}}{48 D}+\frac{P L}{4 U}$

$U=\frac{G(h+c)^{2} b}{4 c}$

where

$G$ is the shear modulus of core mat;

$E$ is the modulus of face sheet;

$t$ is the face sheet thickness;

$c$ is the core mat thickness.

In accordance with the standard test method mentioned before, at least five specimens are required to obtain average results. The load was measured using a load cell attached to the ram and the displacement was measured at the mid span position with the aid of a video gauge. A schematic of the 3PBT is shown in Fig. 2. The results obtained from experimental testing including the ultimate flexural strength, the flexural modulus and the flexural failure strain were used as input data in the finite element model.

Table 1 Configurations of sandwich composites used in 3PBT

\begin{tabular}{lll}
\hline Material & Number of layers & Thickness $(\mathrm{mm})$ \\
\hline CSM1 & 5 (2 skin and 3 core mat $)$ & Skin $=2.25$, core mat $=1.15$ \\
CSM2 & 3 (2 skin and 1 core mat $)$ & Skin $=2$, core mat $=4$ \\
CSM3 & 3 (2 skin and 1 core mat $)$ & Skin $=2$, core mat $=0.75$ \\
\hline
\end{tabular}




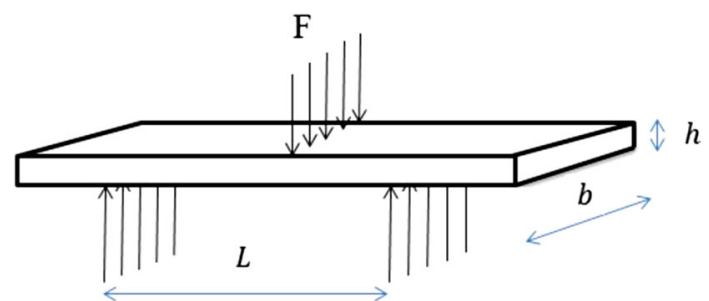

Fig. 2 Schematic representation of 3PBT set up; $L=$ outer span, $b$ and $h$ are width and thickness respectively

\subsection{Fatigue Tests}

The flexural fatigue tests were carried out in displacement control mode. As shown in Fig. 3, the tests were performed under a sinusoidal form of cyclic loading with a frequency of $0.5 \mathrm{~Hz}$ and the stress ratio of $R=0.1$ (the ratio of the minimum stress to the maximum stress in one cycle) according to ES BN ISO 13003 standard. Four stress levels of $\sigma_{\max }$ (applied maximum stress) i.e. 80, 70, 60 and $50 \%$ of ultimate static bending stress, were selected for the fatigue tests. A total of 5 samples were tested at each stress level. The fatigue test was carried out with a constant strain level until failure. An autonomous data logger connected to the load cell in the fatigue rig recorded the load-time response during tests, at a frequency of $100 \mathrm{~Hz}$ enabling to observe the variation of load versus time (cycles) during the fatigue test.

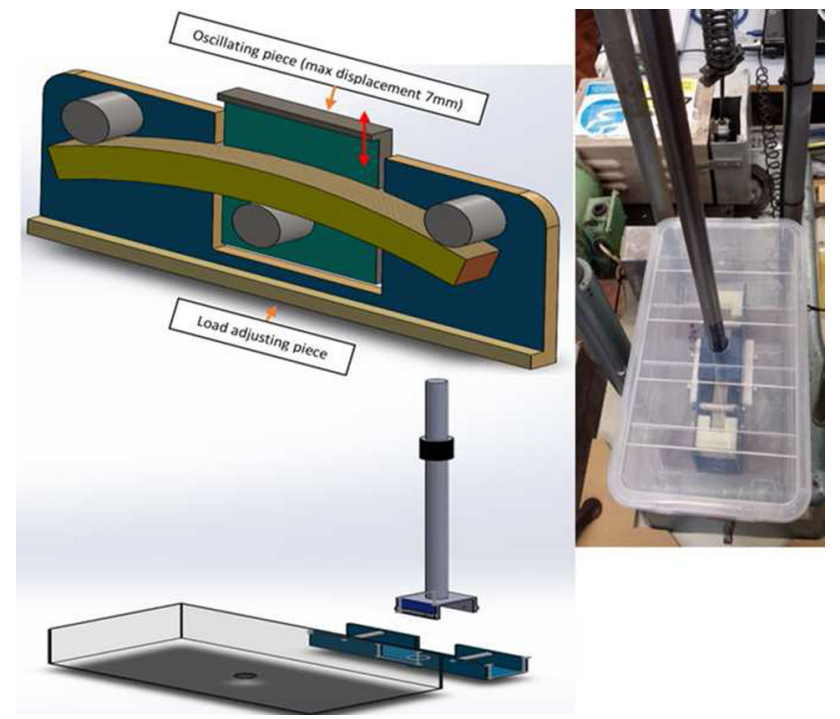

Fig. 3 Fatigue test rig

\section{Finite Element Modelling of 3PBT}

Numerical simulations were performed using the ANSYS 15 finite element software. The simulations of 3PBT were conducted using modelled samples with physical and mechanical properties given in Table 2 . These values have been found theoretically [8, 10]. Figure 4 shows the boundary conditions used. The two supporting points at either end of the parallel sided specimen are fixed for translation at $z=0$ and a flexural load is applied opposite to the supporting points. Such a model offers the potential for numerical investigations of the effect of material parameters, but they must initially be validated with respect to experimental data. A 3D model of all composite specimens was created by using an element type SHELL 4 node 181 which is a three-dimensional four node element. For this element, the six degrees of freedom at each node are translation in three $(x, y, z)$ directions and rotations about these axes. In Fig. 5, the location of the element nodes, the coordinate system and the geometry are illustrated.

Convergence tests were conducted on a model of the sample. An appropriate number of elements were obtained by increasing mesh density step-by-step. The Von-Misses stress was the criterion for the convergence tests. Figure 6 shows the results of the convergence analysis. It shows that convergence is obtained with the use of approximately 20,000 or more elements.

\section{Results and Discussion}

\subsection{Results of 3PBT}

Flexural strength and modulus of the composites have been determined. Typical stress-strain curves, obtained from a 3PBT, are presented in Fig. 7. The mechanical properties are well matched with the results reported in the literature $[11,12]$. From the results, it can be observed that the general trend of composites in the 3PB test for all samples is very similar. Initially, the load increases proportionally with displacement; the materials show a linear elastic behaviour until to the point of fracture under the maximum load.

From visual observation of the test, beyond the point of fracture crushing of the face sheets associated with the distortion of the PVC core mat is observed. This wrinkling effect is obvious in sandwich composite structures with low density core mat [13]. Further loading leads to face sheet failure and this phenomenon is followed by a decrease in the bending load. Finally, the structure collapses due to the propagation of cracks at the face sheet/core interface and 
Table 2 Mechanical properties of sandwich composite constituents

\begin{tabular}{lcc}
\hline Mechanical properties & CSM (face sheet) & PVC foam (core mat) \\
\hline Elastic modulus, $E(\mathrm{GPa})$ & 7.8 & 2.34 \\
Poisson's ratio, $v$ & 0.33 & 0.39 \\
Density $\left(\mathrm{kg} / \mathrm{m}^{3}\right)$ & 1700 & 1205 \\
\hline
\end{tabular}

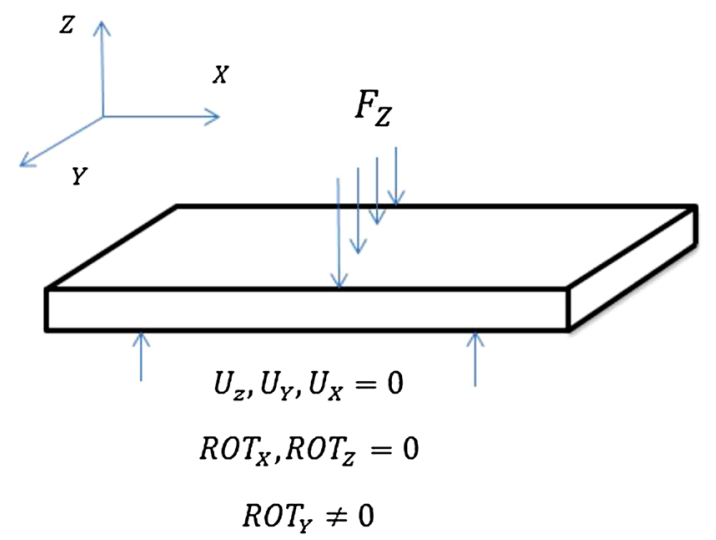

Fig. 4 Load application and boundary conditions for 3PBT. $F_{z}$ is the load applied in $z$ direction; $U_{x}, U_{y}$ and $U_{z}$ are the displacements in the $x, y, z$ directions; $R O T_{x}, R O T_{y}$ and $R O T_{z}$ are the rotations around $x, y$, $z$

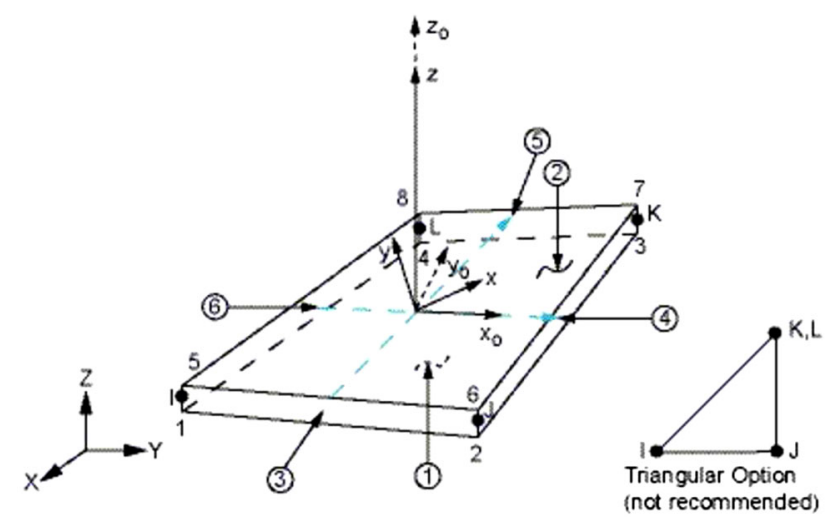

Fig. 5 Element geometry of SHELL 4 node 181 (from ANSYS manual)

shear fracture of the core mat. The reason why the cracks start to propagate at the interface of the face sheet and core is explained by differences in Young's moduli of the face sheet and core mat materials. As shown in Fig. 7, among all types of sandwich composites, the thinnest one (CSM3) shows the highest flexural modulus, flexural strength and deformation. Inferior mechanical performance of sandwich composites (CSMs) with higher number of layers and thickness (CSM1 and CSM2) can be attributed to bad face sheet /core adhesion which can induce a premature failure of the sample, due to crack initiation at the interface between the two constituents $[14,15]$.

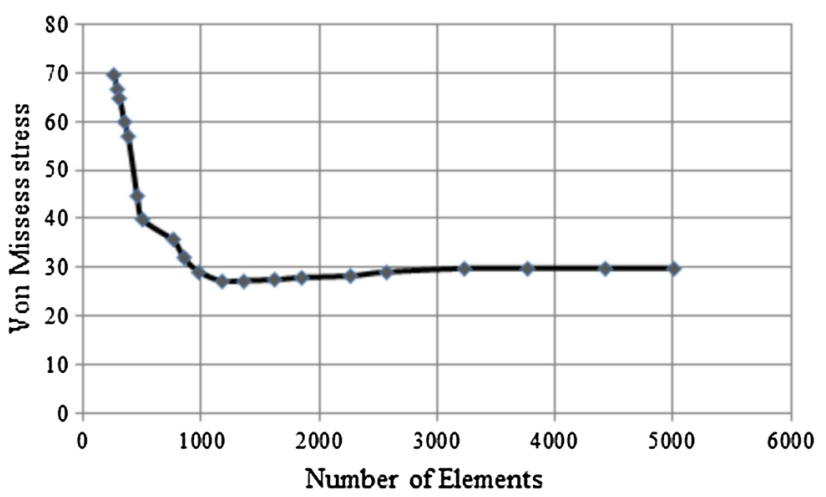

Fig. 6 Convergence test based on the Von-Misses stress

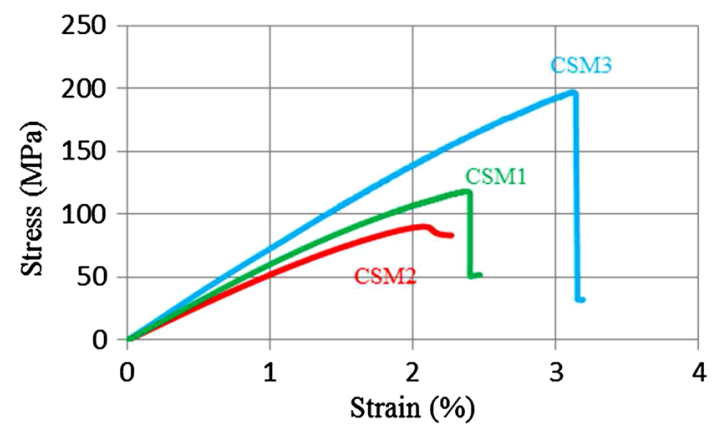

Fig. 7 Stress-strain curves for sandwich composite structures

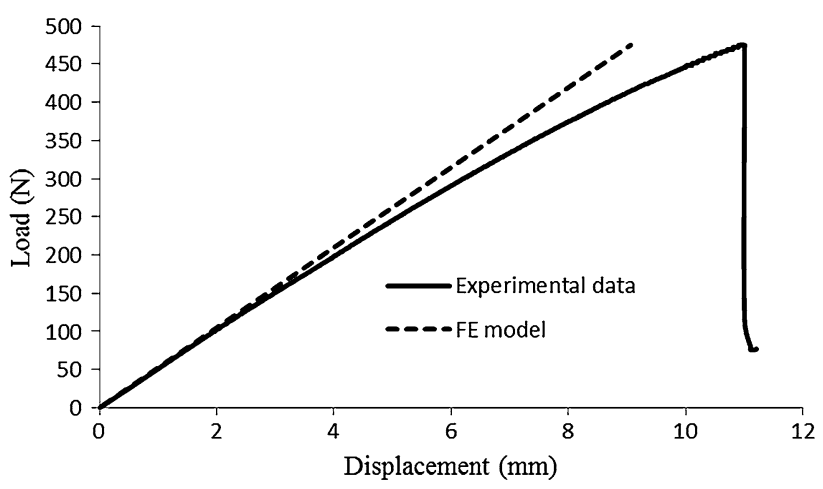

Fig. 8 Experimental/numerical results for 3PB test of CSM3 sample

\subsection{Finite Element Analysis of 3PBT}

Figure 8 shows the results from ANSYS simulations of 3PB test and experimental data. An appreciable compatibility between the numerical analysis and the experiments 


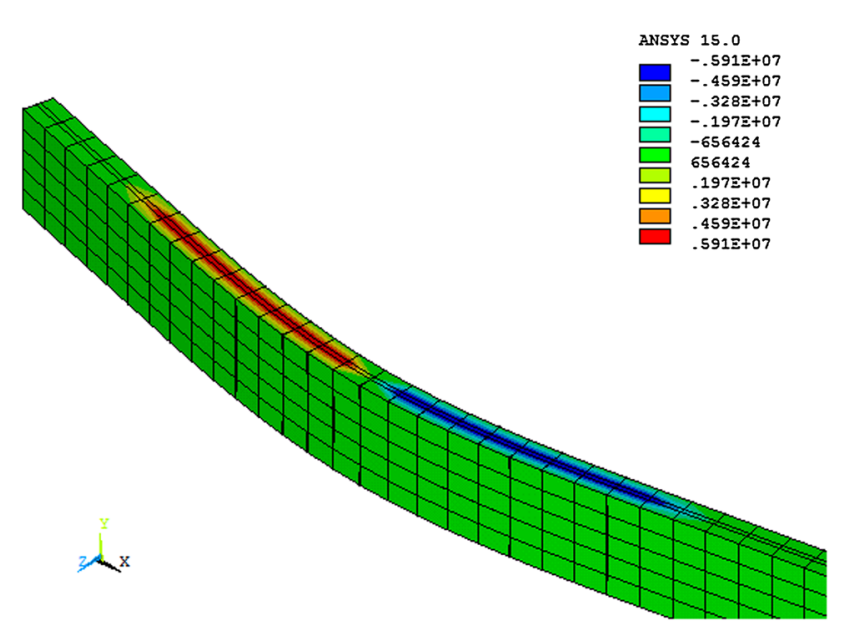

Fig. 9 Shear stress distribution in the $z$ direction (CSM 3)

at lower deformations is noticed. Only representative load displacement curves are shown (CSM3). Deviations at high loads highlight the effects of defects created during the test. Figures 9 and 10 show stress distributions in the applied load direction for three point bending test, just before the point of fracture in the load-deflection curve. As it can be seen for sandwich composite structures, the higher shear stress values are reached in the core mat, while bending stress values are higher in the face sheets. The bending stress is maximum in the outer surface in the form of compression and tension in concave and convex side of test specimen, respectively. A comparison of mechanical properties between ANSYS data and experiments is given in Table 3.

\section{3 $S-N$ Curves}

Fatigue behaviour can be attributed to ultimate flexural strength of the composite materials by linear law relationship between stress magnitude $\left(\sigma_{a}\right)$ and fatigue life of the form

$\sigma_{a}=\sigma_{\mathrm{ufs}}-b \log N_{\mathrm{f}}$

where

$\sigma_{\mathrm{ufs}}$ is ultimate flexural strength of material

$N_{\mathrm{f}}$ is the number of cycles to failure

$b$ is a constant (can be determined from $S-N$ curves).

In order to determine the life time diagram, five specimens were tested at a minimum of four separate levels of imposed stress. For measuring the monotonic strength for the selected test method, five specimens were tested under 3PB monotonically to failure. The stress-life fatigue test results along with the corresponding linear law line fits, as

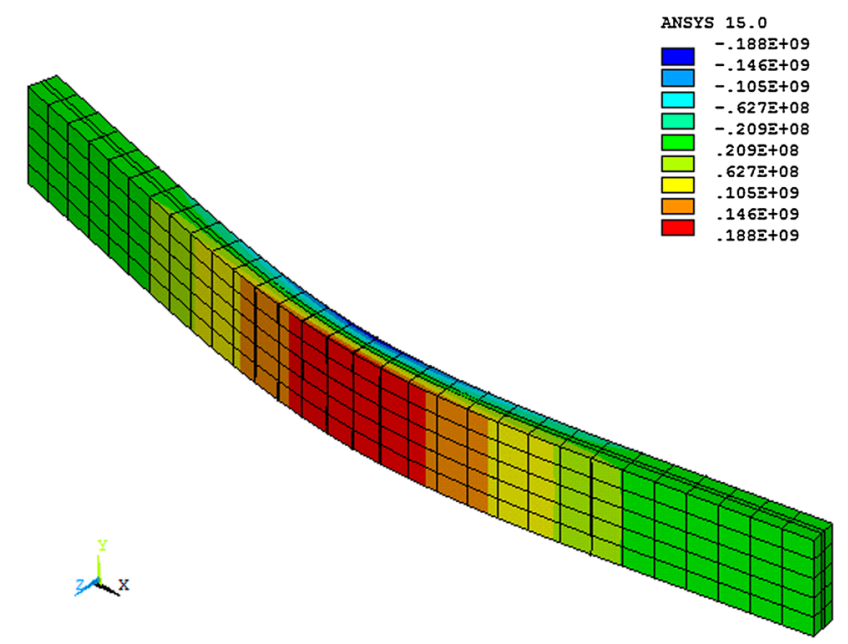

Fig. 10 Bending stress distribution in the $z$ direction (CSM 3)

per above equation are shown in Fig. 11, in which each data point indicates one valid flexural fatigue test.

Comparing the $S-N$ curves obtained in air with those obtained in seawater shows that the seawater influences the fatigue behaviour. For the sandwich composites studied in this paper, at a particular cyclic stress, the fatigue life in air is higher than that in seawater but the difference is not large. The safe region is below the $S-N$ curves and these composites would be able to support the fatigue loads, while above the $S-N$ curve they would fail by fatigue. In order to predict the fatigue life of the composites at other stress levels not included in the current tests, equation (5) is used. The linear regression fitting parameter was determined as $b=30.111$. This is an indicator of the strength loss in time. The loss in strength is lower for the sandwich composites when tests are performed in seawater. A region with a confidence level of $90 \%$ for the fitted curve was established. This means that $N$ corresponding to each stress level has a precision of $90 \%$ in predicting the life of composites.

In order to estimate the damage evolution and to better understand how alternating loads in seawater influence the composites, the fatigue tests have been interrupted after different periods of time proportional to the fatigue life time at a stress level of $80 \%$ of UFS. Then, quasi-static 3PBT was performed to failure in order to determine the residual flexural strength as illustrated in Fig. 12. This is an indication of the reduction in stiffness as a result of cyclic loads. Even after $70 \%$ of the fatigue life, the residual strength is not reduced which is associated to residual stress release.

Stiffness degradation of composites during fatigue tests is also confirmed by the output from the fatigue rig. These data show diagrams of load versus time. As shown in Fig. 13, the stiffness of composites under cyclic loading 
Table 3 Experimental/ numerical comparison of mechanical properties

\begin{tabular}{|c|c|c|c|c|c|c|c|c|}
\hline \multirow[t]{2}{*}{ Material } & \multicolumn{2}{|c|}{$E(\mathrm{GPa})$} & \multicolumn{2}{|c|}{ Flexural strength (MPa) } & \multicolumn{2}{|c|}{$\Delta(\mathrm{mm})$} & \multicolumn{2}{|c|}{$\partial(\mathrm{MPa})$} \\
\hline & FEA & Exp & FEA & Exp & FEA & Exp & FEA & Exp \\
\hline CSM1 & 6.8 & 6.07 & 116 & 118 & 3.4 & 5 & 4.9 & 4.3 \\
\hline CSM2 & 5.9 & 5.04 & 93 & 90 & 2.8 & 4.4 & 3.4 & 3.2 \\
\hline CSM3 & 7.8 & 7.46 & 188 & 197 & 9 & 10.9 & 5.9 & 5.4 \\
\hline
\end{tabular}

decreases with increasing time (loading cycles). This means that the mechanical performance of composites degrades gradually during fatigue test. With increasing number of cycles, the load decreases but near the very end of the fatigue cycle the load decreases very sharply indicating that the damage of test specimen is catastrophic. There is no significant reduction in mechanical performance during the first 40,000 s (corresponding to 20,000 cycles) but the stiffness drops suddenly at 40,000 s. After this the failure of the composite occurs in a few cycles. The catastrophic failure of the test specimen was explained by Kulkarni [16]. The catastrophic failure is due to the failure of the core mat by shear.

As shown in Fig. 11, the number of cycles increases as the fatigue stress level decreases. At all stress levels, the mechanism and modes of fatigue failure were the same as for quasi-static 3PB. Damage initiation occurs in the compression and tension side within a small distance away from the loading roller. Damage can be observed in the sample as stress whitening due to matrix cracking and crazing. At higher number of cycles or at an increased stress level, the damage progressed out from the loading area and it extended through the thickness and final failure occurred on both sides.

\section{Conclusions}

The fatigue performance of sandwich composites was investigated in air and in seawater. Finite element simulation results were validated by three point bending tests and fatigue tests.

The results of 3PBT show that decreasing the thickness of the core and the number of layers will result in better mechanical properties (i.e. less chance of failure in bending).

From macroscopic observation of the failed specimens under quasi-static 3PBT, de-bonding, fibre pull-out and matrix cracking are the typical failure modes observed.

The comparison of the experimental results and the simulation results shows that there is good agreement between finite element analysis and experimental results at lower loads. Deviations at high loads highlight the effects of defects created during the test.

Fig. 13 Load versus time at a stress level of $80 \%$ of UFS 
Acknowledgments This work was supported by the Engineering and Physical Sciences Research Council (EPSRC) [Reducing the Costs of Marine Renewables via Advanced Structural Materials (ReC-ASM), grant number EP/K013319/1].

\section{Compliance with Ethical Standards}

Conflict of interest On behalf of all authors, the corresponding author states that there is no conflict of interest.

Open Access This article is distributed under the terms of the Creative Commons Attribution 4.0 International License (http://crea tivecommons.org/licenses/by/4.0/), which permits unrestricted use, distribution, and reproduction in any medium, provided you give appropriate credit to the original author(s) and the source, provide a link to the Creative Commons license, and indicate if changes were made.

\section{References}

1. Belouettar S, Abbadi A, Azari Z, Belouettar R, Freres P (2009) Experimental investigation of static and fatigue behaviour of composites honeycomb materials using four point bending tests. Compos Struct 87(3):265-273

2. Borsellino C, Calabrese L, Valenza A (2004) Experimental and numerical evaluation of sandwich composite structures. Compos Sci Technol 64(10-11):1709-1715

3. Di Bella G, Calabrese L, Borsellino C (2012) Mechanical characterisation of a glass/polyester sandwich structure for marine applications. Mater Des 42:486-494

4. Russo A, Zuccarello B (2007) Experimental and numerical evaluation of the mechanical behaviour of gfrp sandwich panels. Compos Struct 81(4):575-586
5. Branner K (1995) Capacity and lifetime of foam core sandwich structures. Technical University of Denmark, Lyngby

6. Davalos JF, Qiao P, Xu XF, Robinson J, Barth KE (2001) Modeling and characterization of fiber-reinforced plastic honeycomb sandwich panels for highway bridge applications. Compos Struct 52(3-4):441-452

7. Mullin JV, Knoell AC (1970) Basic concepts in composite beam testing. Mater Res Stand 10(12):16

8. Hollaway LC (1994) Handbook of polymer composites for engineers. Woodhead Publishing, Cambridge

9. BSI. BS EN ISO 14125:2011 - Fibre-reinforced plastic composites. Determination of flexural properties. British Standard Institution, 2011

10. CES Edupack. Granta Design Limited, 2014

11. Christiansen AW, Lilley J, Shortall JB (1974) A three point bend test for fibre-reinforced composites. Fibre Sci Technol 7(1):1-13

12. Mines RAW, Alias A (2002) Numerical simulation of the progressive collapse of polymer composite sandwich beams under static loading. Compos Part A 33(1):11-26

13. Weissman-Berman D (1992) Marine sandwich structures (part ii of ii). SAMPE 28:9

14. Gregory JR, Spearing SM (2005) Constituent and composite quasi-static and fatigue fracture experiments. Compos Part A 36(5):665-674

15. Mouritz AP, Thomson RS (1999) Compression, flexure and shear properties of a sandwich composite containing defects. Compos Struct 44(4):263-278

16. Kulkarni Nitin, Mahfuz Hassan, Jeelani Shaik, Carlson Leif A (2003) Fatigue crack growth and life prediction of foam core sandwich composites under flexural loading. Compos Struct 59(4):499-505 